\title{
Cholesteric-type cellulosic structures: from plants to applications
}

\section{Ana P. C. Almeida, João P. Canejo, Pedro L. Almeida \& Maria Helena Godinho}

To cite this article: Ana P. C. Almeida, João P. Canejo, Pedro L. Almeida \& Maria Helena Godinho (2019) Cholesteric-type cellulosic structures: from plants to applications, Liquid Crystals, 46:13-14, 1937-1949, DOI: 10.1080/02678292.2019.1640904

To link to this article: https://doi.org/10.1080/02678292.2019.1640904

曲 Published online: 24 Jul 2019.

Submit your article to this journal $\pi$

Џll Article views: 187

Q View related articles $\sqsubset$

View Crossmark data \lceil 


\title{
Cholesteric-type cellulosic structures: from plants to applications
}

\author{
Ana P. C. Almeida $\left(\mathbb{D}^{a}\right.$, João P. Canejo $\left(\mathbb{D}^{a}\right.$, Pedro L. Almeida $\left(\mathbb{D}^{\mathrm{a}, \mathrm{b}}\right.$ and Maria Helena Godinho $\mathbb{( D}^{a}$
}

${ }^{a}$ CENIMAT/I3N, Departamento de Ciência dos Materiais, Faculdade Ciências e Tecnologia, FCT, Universidade Nova de Lisboa, Campus da

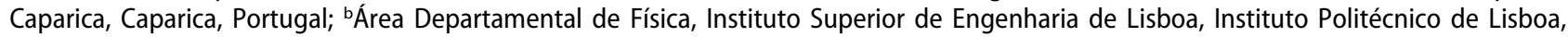
Lisbon, Portugal

\begin{abstract}
The structural support of plant cells is provided by the cell wall, which major load-bearing component is an array of hierarchical orientedhierarchical-oriented cellulose nano-, micro- and meso-structures of cellulose microfibrils. Cellulosic structures can respond to humidity changes by expanding or shrinking and this allows, for example, the dispersion of seeds. Previous studies have shown that nanorods, extracted from cell walls, can generate lyotropic liquid crystals that are at the origin of solid cholesteric-like arrangements. Not only photonic films, but also right and left helical filaments, anisotropic films with the ability to bend back and forth under the action of a moisture gradient at room temperature, are some of the materials that were produced from cellulose liquid crystal systems. This work is a review that focus on liquid crystalline-based structures obtained from cellulosic materials and how small perturbations on their structures affect significantly the response to external stimulus and interactions with the environment. Special emphasis is given to cholesteric-like organization of cellulose structures existing in plants, which are an inspiration for the production of the next generation of soft interactive materials.
\end{abstract}

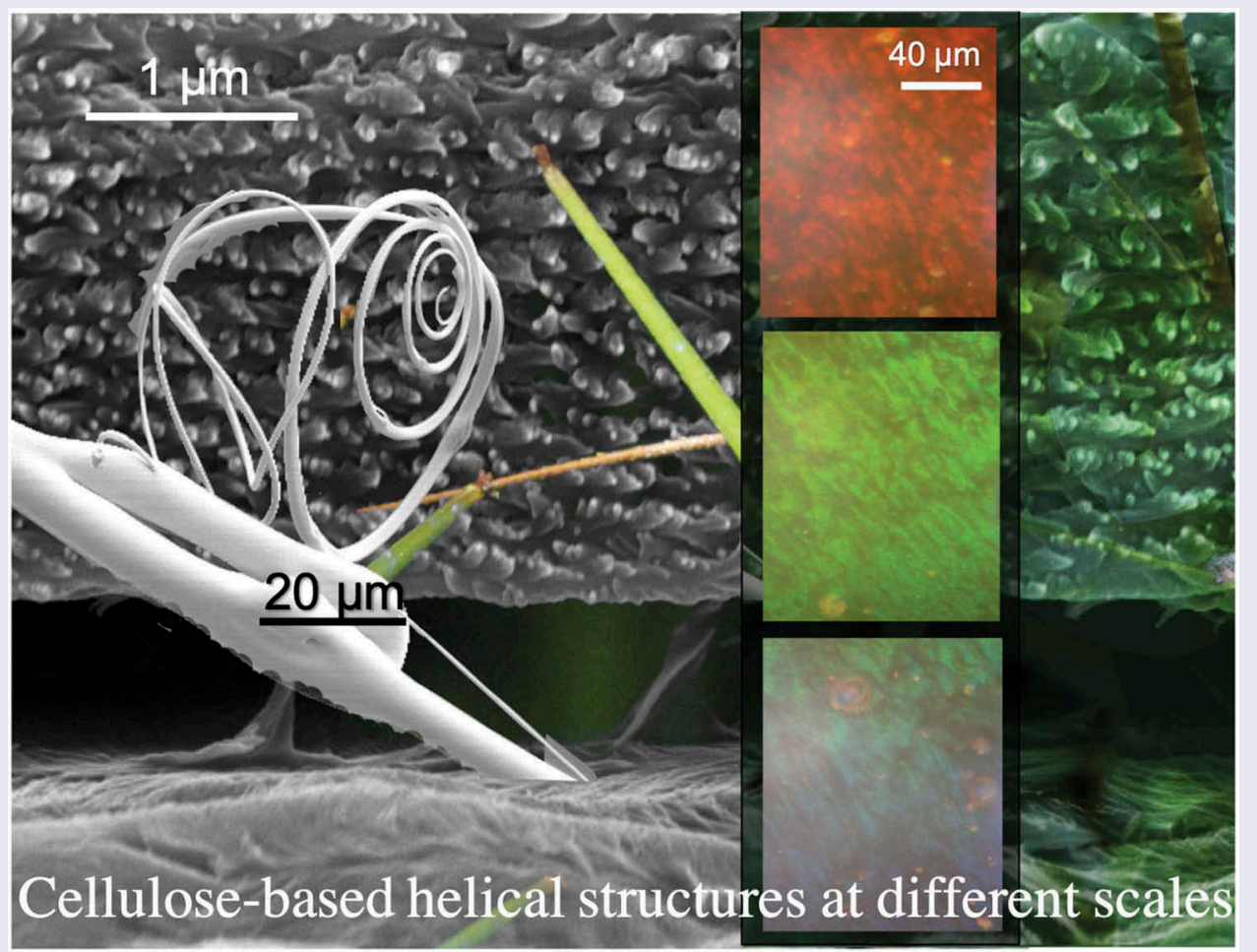

\section{ARTICLE HISTORY}

Received 8 May 2019

\section{KEYWORDS}

Cellulose; cholesteric phases; cellulose-based lyotropic systems; thermotropic cellulosic systems; photonic materials; responsive cellulose-based materials

\section{Plants cholesteric-type structures}

Plants contain, at different scales, anisotropic cellulose structures that are responsible, for instance, for colour or/and movement. Most of the colors exhibited by plants are due to pigments and dyes molecules such as chlorophyll a. These pigments fade with time and are ephemeral. Plant extracts used as dyes, such as saffron or indigo

CONTACT Maria Helena Godinho mhg@fct.unl.pt 
are damaged by light [1]. Another way of plants to present lively and more attractive colours is achieved by the interaction of light with ordered structures at the micron and nanoscale [2]. The structural colour observed in some plants, such as the white colour of cotton (Figure 1(a)), results from optical scattering of randomly distributed microfibres [3]. This effect was reproduced with white electrospun randomly distributed cellulose-based micro/nanofibres (Figure 1(b)). Other colours result from the interaction (reflections) between the incident light and naturally occurring ordered structures [4,5]. Well-documented examples are the diffraction gratings of the Queen of the Night tulip (Figure 1(c)) and in ordered structures existing in Selaginella (Figure 1(d)) [2], which were replicated with cellulose derivatives [6]. Besides structural colours, the pigments in plants may contribute with additional colours, due to selective light absorption. The colours resulting from ordered structures differ with the viewing angle or lighting geometry, which means that they present iridescence [7], like the interference colours seen in thin soap films or bubbles $[8,9]$.

Cellulose is produced mainly by plants and the chiral centres of the polymer backbone are normally associated with the formation of a liquid crystalline cholesteric phase (also known as chiral nematic phase) [10,11], as schematically represented in Figure 1(e). The cholesteric phase is present in lyotropic as well as in thermotropic systems, which are obtained from cellulose-based materials, and a pallet of structural colours can be obtained [12]. The cholesteric structure is characterized by the sense and the value of the pitch $(\mathrm{P})$ of the helical configuration [13,14], as shown in Figure 1(e). These helical structures are responsible for the cholesteric iridescence of the material, and its selective reflection depends on the viewing angle, the wavelength of the incident light and the sense of its circular polarization [15].

A cholesteric structure presents a reflected wavelength value $(\lambda)$ which is conditioned by the length of the helical pitch $(P)$ and the average refraction index $(\bar{n})$ of the material, according to de Vries expression [16]

$$
\lambda=\bar{n} P(\sin \theta)
$$

considering $\theta$ as the angle between the propagation of the incident light and the direction perpendicular to the cholesteric layers. Therefore, at a normal angle of incidence $(\theta=\pi / 2 \mathrm{rad})$, the wavelength of the reflected light is directly proportional to the cholesteric helical pitch $[17,18]$. From the white disordered cotton structure, cholesteric vivid, iridescent colours can emerge if anisotropic structures are isolated and self-assembled (Figure 1(f)). The values of the cholesteric pitch, in the range of the wavelength of the visible light [12] determine the colour of the samples. Moreover, for normal light incidence, circularly polarized light having the same handedness of the helix is completely reflected, whilst a circularly polarized light with opposite handedness is completely transmitted [14,15].

In the 1960s, Bouligand and later on, Neville $[19,20]$, implemented experiments that evidenced that the optical properties, and the structures observed by Scanning Electron Microscopy (SEM), of the cuticles of some beetles and other biological systems such as plants. They found that the structures observed were somehow analogous to what was known for cholesterics [21,22].

Bouligand, was responsible for far-reaching research on cholesteric structures, showing meticulous threedimensional prototypes and drawings of their textures and morphology (Figure 2(a)) and other living beings

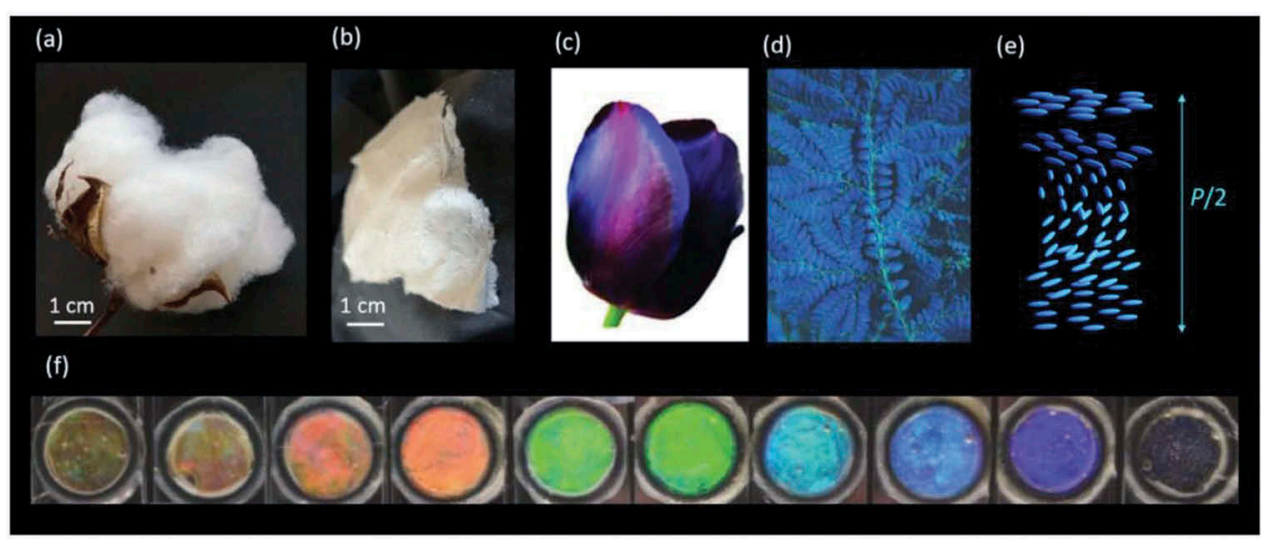

Figure 1. (Colour online) Cellulosic structural colours. (a) Cotton flower, (b) Electrospun cellulose-based non-woven membrane (c) Tulip Queen of the Night flower [6] Copyright @ 2013 WILEY-VCH Verlag GmbH \& Co. KGaA, Weinheim, (d) Blue iridescence genus Selaginella plant reproduced with permission from [2], (e) Scheme of a cholesteric structure, where $P / 2$ is half the length of the structure's pitch, (f) Macroscopic images of HPC/water mesophases with different concentrations (low to high HPC concentration in water, from left to right) reproduced under Creative Commons Attribution 4.0 International License [12]. 
(a)

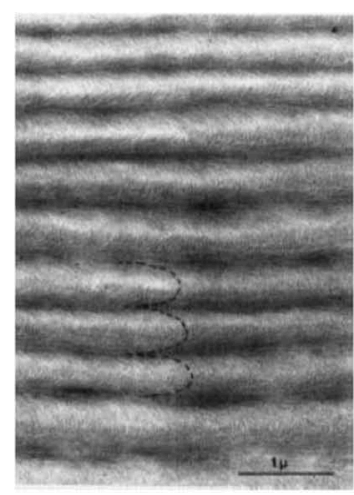

(b)

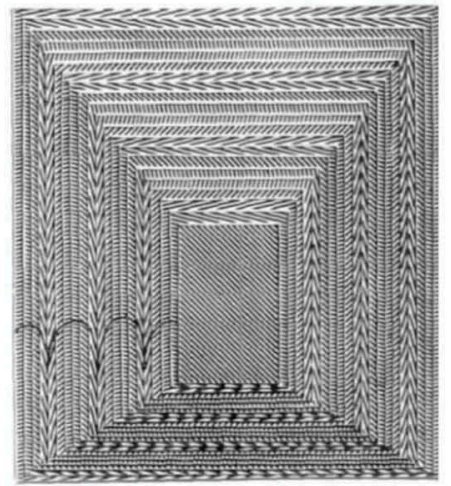

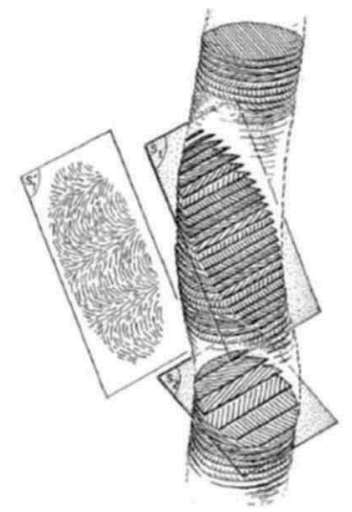

(d) (c)

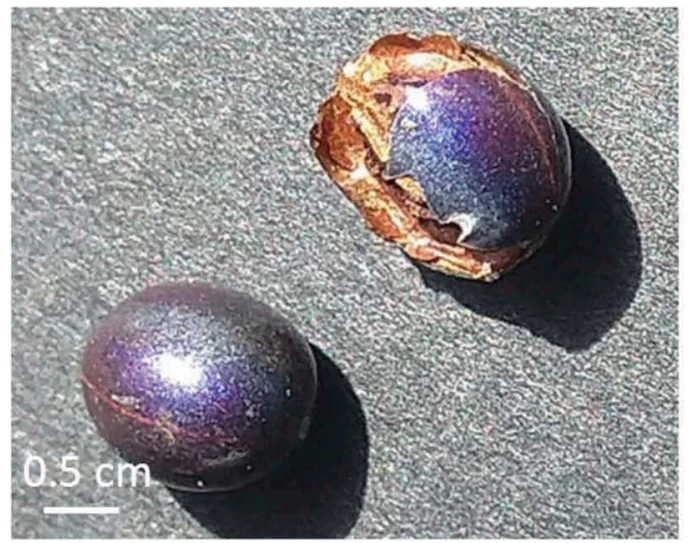

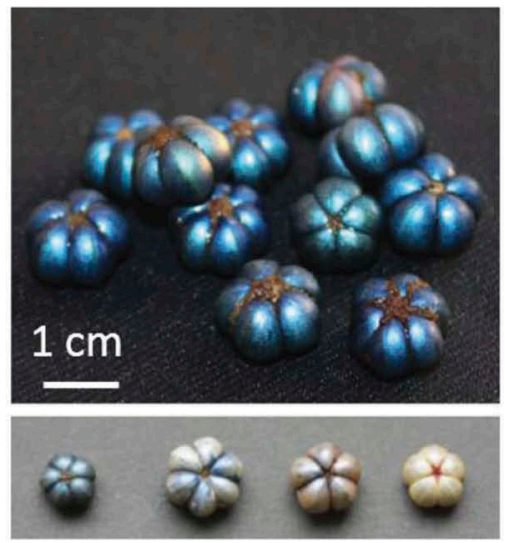

Figure 2. (Colour online) Cellulose-based helicoidal structures. (a) Periodic arcs shaped during cellular differentiation [32], (b) Bouligand structure showing a stacking of layers in which the lines in each layer are rotated $30^{\circ}$ from the previous layer. Cylindrical model, cut by oblique planes, idealized by Bouligand to visualize the formation of arcs in a cholesteric structure [27]. (c) Picture of Pollia condensata fruit, (d) Fresh fruits of Margaritaria nobilis. Fruits at successive stages of desiccation, from left to right, fully hydrated to dry, respectively. The average dimension of the fruits is about $1 \mathrm{~cm}$. Reproduced from [31] with permission of ROYAL SOCIETY in the format Republish in a journal/magazine via Copyright Clearance Center.

[22-25]. Bouligand evidenced the similarity existing between the molecular orientation of the molecules in the cholesteric phase and observed the orientation of microfibrils in plant structures (Figure 2(b)).

The dimension of the constituents of the helicoidal structures might range from the nanoscale to a few micrometres. Bouligand proposed a model in which a set of fibrils with arcs is arranged in space with a certain periodicity. In this model, the fibrils had an axial symmetry and an oblique section of these fibril arrangement results in a series of arcs whose direction of concavity reverses on either side of the trace of the axis. Whatever the direction of an oblique cut, the series of arcs obtained, have all the same sense of concavity. These particular geometrical arrangement is found in several very different biological materials with different functions [26]. Bouligand described that a regular lamellar structure is revealed by sectional examination of a large number of biological systems such as plants and beetles. The lamellae containing fibres arranged in a characteristic way were observed by SEM. Each lamella observed in an oblique section is apparently composed by fibres arranged according to arcs. In fact, he verified the lack of fibrils curling (Figure 2(b)) in the thickness of the lamellae; this aspect is an illusion due to the fact that the filaments are parallel to each other, but their orientation gradually changes, and in each successive layer the fibrillar direction is turned in a small angle about an axis perpendicular to the planes. These bow-shaped geometries are characteristic of cholesterics (Figure 2(b)) [27].

Bouligand stated that the twisting of fibrils in one direction, for a certain number of biological materials, could arise from the helical or double helical configurations of the biological macromolecules. He attributed the formation of these organized solid structures to 
liquid state secretions of plants that self-assembly in an ordered manner. The existence of ordered fluids and possibly ordered liquid secretions could provide an interesting mechanism in morphogenesis for certain fibrous networks [22].

Plants produce cellulose using enzymatic processes. The cellulose molecules are assembled into fibrils that are then arranged into larger entities named microfibrils, the units that form fibres [19]. These cellulose fibres form the structures that allow the plants to create skeletons. The orientation that the microfibrils assume within the cellulose fibres depends on numerous factors, but the existence of an helicoidal morphology is vastly prevalent within the plantae in very different plant tissues and purposes [20,28]. In 1993, Graham et al. [29] observed iridescent colours in two ferns, Danaea nodosa and Trichomanes elegans. The authors found that, in the case of the Danaea nodosa, the displayed colours are due to the presence of layers of microfibrils of cellulose in the adaxial epidermis that forms a helicoidal structure. Later, in 1996, Gould et al. [30] studied plants originated from the Malaysian rain forest. These plants possess iridescent leaves and the authors showed that two species, Diplazium tomentosum BI. (Athyriaceae) and Lindsaea lucida BI. (Lindsaeaceae), have a helicoidal structure in the outermost cell wall layer of the adaxial epidermis (in the case of the Diplazium tomentosum) and of the abaxial and adaxial epidermis (in the case of the Lindsaea lucida). The authors proposed that these structures are responsible for the reflected blue and green light, characteristic of these species, due to constructive interference of the reflected light.

Beside leaves, also fruits display iridescent structural colours. In 2012, Vignolini et al. [5] showed that the iridescent blue colour exhibited by the fruit of the Pollia condensata (Figure 2(c)) is due to the presence of thickwalled cells in the epicarp forming a helicoidal structures corresponding to the orientation of cellulose microfibrils oriented in such a way that a helix is formed. One clear indication that the reflected colour is structural (rather than due to pigmentation) is the observation that the colour does not fade with time; the authors reported that one of these fruits collected in the 1970 decade, retains until now a bright colouration. This work surprisingly reports that the Pollia condensata fruit reflects light with both left-handed and right-handed circular-polarized light. More recently, in 2016, Vignolini et al. [31] studied the fruit of the Margaritaria nobilis. This fruit has a green-blue colour when fresh and a pearlescent colour when dry (Figure 2(d)). It was observed that the transition from the fresh to the dry state, and vice-versa, is accompanied by the corresponding colour change, indicating that the process by which the colour is obtained in each state is reversible. The authors found that the exhibited colours have a structural origin and do not depend on the presence of pigments. This work purposes that the described optical behaviour is due to the presence of a helicoidal structure in the cell wall of the pericarp cells. When the fruit is dry, the seeds shrink, creating a layer of air between the seeds and the endocarp, stopping light absorption and, decreasing the contrast. When the fruit is hydrated, the expansion of the seeds eliminates the layer of air and the seeds contact with the endocarp, promoting the appearance of the blue-green colour. These examples highlight the macroscopic effect that can be observed (structural colour) when cellulose microfibrils are present, in a much smaller scale with a periodical helicoidal structure.

\section{Cholesteric-type structures at different scales - from molecules to the macroscale}

Cholesteric-like structures, described in detail in the previous section, can be found in plants across a wide range of scales.

In 1951, Ranby described the production of colloidal suspensions of cellulose fibres obtained by acid hydrolysis of cellulose [33]. One of the designations proposed for cellulose nanocrystals, prepared by for cellulose nanocrystals, prepared by acid hydrolysis, were cellulose nano crystals (CNCs). These crystals are chemically and biologically inert [34]. In the beginning of the 1950s, transmission electron microscopy (TEM) observations performed by Mukherjee and Woods showed that these cellulose fibrils have a crystallographic structure similar to the cellulose fibres $[35,36]$ used in its production. This type of nanocellulose is prepared by separation of the crystalline regions of semi-crystalline cellulosic fibres and of removal of the polysaccharides of the surface of the fibre followed by removal of the amorphous regions.

The observation of a birefringent phase originated by a suspension of cellulose crystallites obtained by acid hydrolysis was first reported by Marchessault et al. in 1959 [37].

The process of acid hydrolysis involved in the production of CNCs is discussed in the comprehensive review made by Klemm et al. [38] concerning nanocelluloses and by other groups, such as Ruiz et al. [39] or Habibi et al. [34]. These works mention the fact that, during acid hydrolysis, the removal of amorphous cellulose regions is favoured compared to the crystalline regions that have higher resistance to chemical attack [38].

Later, in 1992, Revol et al. reported the formation of cholesteric helical structures in suspensions of cellulose 
nanoparticles. In this work, the team describes how a dispersion, in water, of cellulose microfibrils selfassembles above a critical concentration in order to create a phase that is chiral nematic liquid crystalline [40]. Concerning these cholesteric suspensions, one of the most important observations is the fact that cholesteric helix is always left-handed [38], unlike what happens for cholesteric phases of cellulose derivatives where right-handed and left-handed structures can be observed.

Later, in 2001, Fleming et al. [41] demonstrated that the formation of these chiral nematic could be due to the fact that the cellulose microfibrils have a screw-like shape. According to the authors, during packing the fibrils do not align themselves parallel to each other, but as described above (Figures 1(e) and Figure 2(b)).

As mentioned above, cellulose chains that are long and semi-rigid [42], can form liquid crystalline phases [43]. In fact, in 1980 two reports by Chanzy et al. [44] and Navard [45], described the formation of liquid crystalline phases obtained from cellulose solutions. In the following year, Patel [46] also described a similar phase. Later, in 1983 McCormick et al. reported the formation of a lyotropic phase in a solution of cellulose in $\mathrm{N}, \mathrm{N}$-dimethylacetamide [47].

Due to the rigidity of the cellulose main chain, not only cellulose but also its derivatives can form liquid crystalline phases if the conditions are adequate. The hydroxyl groups present in the cellulose macromolecule can be used to promote substitution reactions that originate different derivatives depending on the lateral substituent. In 1976, Werbowyj [48] observed a lyotropic phase-in of hydroxypropylcellulose (HPC) in water for a concentration of approximately $40 \%$ (w/ $\mathrm{w})$, in polymer. When the polymer concentration of approximately $60 \%(\mathrm{w} / \mathrm{w})$, iridescent colours typical of the cholesteric phase were observed. In 1981, Tseng et al. [49] reported that (acetoxypropyl)cellulose (APC) could also form lyotropic phases. Other reports of different cellulose derivatives that form cholesteric phases can be found in the literature [50]. Bheda et al. [51] observed, in 1980 , cholesteric phases in solutions of ethylcellulose in acetic acid for polymer concentrations of almost 50\% (w/w). The critical concentration for the formation of the lyotropic phase depends on the interaction between the side chains of the cellulosic derivative, the solvent used and the rigidity of the cellulose chain. The transition from isotropic liquid to liquid crystal requires mobility of the main chain.

In 1956, Flory [52] proposed a theoretical model to determine the value of the lowest concentration of polymer above which the formation of the liquid crystalline phase occurs, normally referred to as the critical concentration. This model has in consideration the persistence length (q) of the polymeric chains and the value of the Kuhn segment [53].

Besides being able to form mesophases in solution, above a critical concentration, cellulosic derivatives also form thermotropic phases. In 1981, Tseng et al. [49] observed that films of APC exhibit colours when heated. In the same year, Shimamura et al. [54] reported that low molecular mass HPC reflects colour when heated to high temperatures. In 1982, Gray et al. [55] reported the existence of the same effect for the trifluoroacetate ester of HPC. This ability of the cellulosic derivatives to form thermotropic phases is an indication that the side chains act as a solvent (or plasticizer) increasing the mobility of the main chains.

The plant cell wall typically with $0.1 \mu \mathrm{m}$ to $0.3 \mu \mathrm{m}$ thicknesses, serves as a protection for the cells and presents cholesteric order. To provide mechanical strength, the primary cell walls is constituted by cellulose microfibrils of between 500 and 14,000 glucose units [56]. Emons et al. [57] refer in 2000, that one of the possible configurations that the cellulose microfibrils can assume is helical. In 2017, Mitov [56] published a detailed review concerning the presence of cholesteric order in living organisms, including plants.

The advantages for plants of cholesteric structures range from enhanced mechanical properties to the ability to display iridescent colours. From the mechanical resistance point of view, Murugesan et al. [11], developed in 2010 a model based on the Landau-de Gennes theory, in which the cholesteric configuration of the plant cell wall has a better performance against the propagation of fractures by deviating the direction of the propagating crack. In 2017, Tan et al. [58] confirmed the enhancement of the mechanical properties in structures with fibres with an helicoidal alignment. In this work, the authors printed three different types of composites reinforced by helicoidal fibres. Compression tests showed that these structures have higher compressive resistance than other composites reinforced by fibres organized in other configurations. The authors conclude that the increased resistance to compression is mostly dependent on the pitch and the orientation of the optical axis. More recently, Canejo et al. reported on the interactions between stretched liquid crystalline filaments, which modify their topology and geometry, thus being crucial for tuning their mechanical and optical properties [59].

\section{Cholesteric-type structure at the macroscale and movement}

Plants present a wide variety of motions, which are even present in Sclerenchymal tissues of dead cells, 
driven from external stimulus. The slow opening of pine cones [60], the leaf closing of the sensitive plant, Mimosa pudica [61] and the rapid snapping of the Venus flytrap leaf, Dionaea muscipula [62] are some of the movements induced by external elements such as the presence of water or mechanical stimulation. Plant motions, mostly driven by water-swelling or shrinkage of cells inside the tissues, are very different from muscle-driven mechanisms in animals [20]. Plant cells survive significant pressure variations because their cell wall is enclosed with stiff cellulose fibres and other polysaccharides, such as hemicellulose and pectin [63]. The organization of cellulose fibres dictates the changes in the shape of the plant, upon exposed to an external-stimuli. The spatial distribution of the cellulose fibres, between the different constituent materials, determines the shape and complexity of the deformations [64]. The macroscopic movement of the plant is dictated by the organization of cells within the 'mobile' tissue, whereas the mechanical response of the individual cell is determined by the cellulose microfibril stiffening of the cell wall [65].

The organization of plant cells with different mechanical properties may promote stress or movement (or both), when adjacent cells contract (or expand) differently, resulting in accumulation of tension in the tissue [66].

An example of bend-twist motion was observed in a group of plants from the Geraniaceae family. The main characteristics of this family is a beak-shaped fruit that is composed of five mericarps arranged around a central column. The mericarp is composed of a capsule with a fruit inside and a long tapering awn. During the drying of the fruit, the tension is accumulated in the awn cells, as a result of differential contraction of the tissues, and the detachment of awn from the plant is promoted. The coiled awn also responds to changes in the ambient humidity and this mechanism is responsible for the seed self-burial into the ground [66]. An helicoidal structure of a tilted assembly of the cellulose microfibrils, embracing a soft matrix of polysaccharides should dictate the coiling mechanism upon variation of water content (Figure 3(a)). The Erodium is a good representative of this family [67]. In the cells of the Erodium awn, the helix axis (in yellow) forms an angle with the cell axis (in red), promoting a change of the microfibril angle (MFA) between cellulose and the cell axis around the cell (Figure 3(b)). As a consequence of this tilted microfibril helix, compared to the long axis

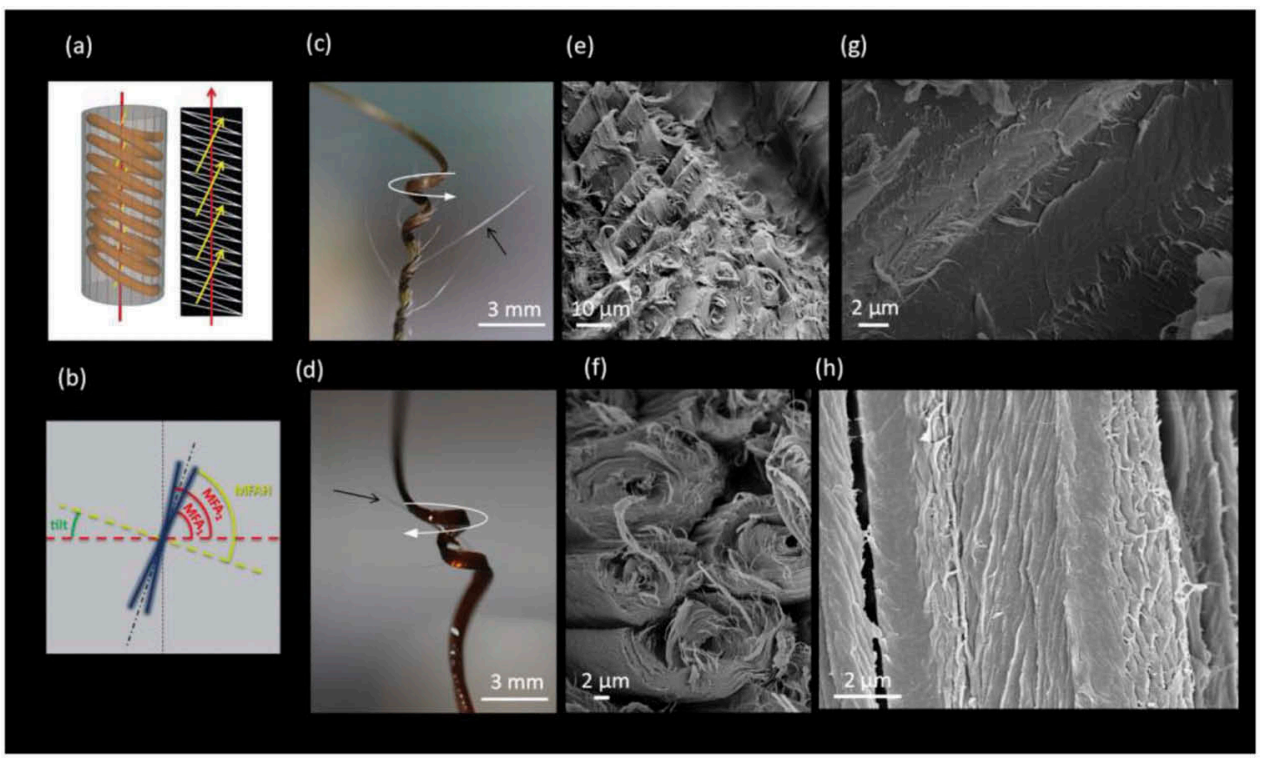

Figure 3. (Colour online) Structure of Erodium cellulose-based awns. (a) Schematic illustration of a cellulose microfibril that is organized in a tilted helix (in orange). The long axis of the cell is represented by a red rod, and the cellulose helix axis by yellow rods and side projection of the cell wall showing that the long axis of the cell (red arrow) lies at an angle to the cellulose helix axis (yellow arrows). (b) Schematic representation of two idealized different microfibril angle (MFA) obtained in relation to the long axis of the cell, represented by MFA 1 and MFA , as the MFA changes around the cell wall in a tilted helix configuration. The tilt, which is the angle between the cellulose helix axis and the long axis of the cell, is indicated in the illustration by a curved green line. MFAH is the angle between the tilted angle and the angle defined by the dotted line (half of the angle between the blue streaks). Reproduced from [68] ๔ 2013 The Authors. New Phytologist @ 2013 New Phytologist Trust. Side views of Erodium awns, dry (c) and wet (d) after swollen in an alkali solution. Reproduced from Ref [71]. With permission from The Royal Society of Chemistry 2019. The arrows indicate the sense of rotation. Hairs, denoted by black arrows, are outside (c) and inside (d) of the helicoidal awns. SEM pictures of fibre cells of the original (e) and (g) and after alkali treatment ( $f$ ) and (h), isolated from the inner layer of the Erodium awns. 
of the cell, a contraction, in a direction perpendicular to the cell long axis, is generated. This contraction causes the cell to simultaneously bend and twist, when the cells dry, resulting in a macroscopic coiling movement $[68,69]$. Other hygroscopic tissues, found in awns of wild wheat, present a layered structure is found responsible for the bending movement of the awn [70]. The layered structure presents soft and rigid constituents, which contract asymmetrically in the presence of water. The cellulose fibrils alignment, in each layer, seems to determine the bending of the awns.

A cellulose-based skeleton was isolated, chemically modified, and used as a template from Erodium awns. The cellulose-based material, which reversibly changes its chirality as a response to variations in the hydration levels, was recently reported [71]. The Erodium righthanded helix (Figure 3(c)) is converted to a left-handed helix after alkali treatment (Figure 3(d)), returning to right-handed structure after drying. The wet structure (left-handed) shows long hairs at the inner surface of the helix, contrary to the dry structure (right-handed), as a consequence of the macroscopic reversal of their chirality. The chemical treatment modified the physical properties of the cells responsible for the movement without affecting the coiling mechanism. The sewing thread-like and circular fibre cells of the pristine awn (Figure 3(e)) after chemical treatment displays wrinkles along the tangential axis and a sinuous-like cross-section (Figure 3(f)). The chemical treatment modified the physical properties of the cellulose network but did not destroy the structure at the nanoscale, preserving their tilted structure [71] (Figure 3(g) and (h)). The curvature and torsion arise from the pristine-tilted arrangement of the cellulosic fibre in the active fibre cells. The inversion of the helix at the macroscale is linked with the stretching of the tilted cellulosic fibre network in diagonal directions. This behaviour is possible because the cellulosic network becomes more permeable to water due to the chemical treatment. The structure imprinted by the plant is always ruling the movement. However, the higher expansion and contraction of the fibre cells, when compared to the non-treated structure, drives the observed inversion of chirality [71].

Solid films prepared from HPC/water anisotropic solutions, showed a behaviour similar to liquid crystal networks. The films responded anisotropically to humidity, as a result of the organization of the rodlike fragments, coupling the orientational order to mechanical strain [72]. The solutions were spread on a glass substrate, with the free surface exposed to air. The cellulose concentration is high enough for the formation of the liquid crystal phase and the alignment of the mesogenic fragments was achieved by sheer with a calibrated Gardner knife moving at a controlled speed (Figure 4(a)). When the films were exposed to humidity the water penetrated the sample promoting its bending around an axis parallel to the shear direction, as shown in Figure 4(a) i), with the free surface on the outside. This behaviour was due to the expansion of the free surface of the film in the direction perpendicular to the director and the decrease of the order parameter in the presence of water [72]. If the glass side of the film was exposed to water vapour, it bended around an axis perpendicular to the shear direction, (Figure 4(a) ii). This behaviour is consistent with a nearly isotropic expansion of the glass side of the film surface due to the presence of water [72].

The design and fabrication of a sandwich-like structure based on a cellulose nanocrystals (CNC) composite, using a simple and inexpensive self-assembled method, was reported by $\mathrm{Wu}$ et al. [73]. This system is composed of flexible polyethylene glycol diacrylate (PEGDA) mixed with CNCs in a uniaxially oriented polyamide-6 (PA-6) film, used as a half-wave retarder in the sandwich structure (Figure 4(b)). The composite photonic film produced presents reversible threedimensional deformation when exposed to humidity and simultaneous shift of the Bragg reflection band (Figure 4(c)). The film bends in the opposite direction of the moisture source. This behaviour is due to the increase of the helical pitch on the side of the film exposed to wet air and consequent asymmetric expansion of the sandwich-structured composite film. This asymmetric expansion is also promoted due to the presence of the PA-6 interlayer that acts as a hydrophobic barrier. The deformational behaviour, that can be twisting and bending of the sandwich structured photonic film in the presence of wet air, is dependent of the cutting angle. The change in the reflected colour was also observed during this deformation process as can be seen in Figure 4(c). The CNC nanocomposite film returned to the initial state when the humidity was removed. This composite device presents a promising potential for the development of humidity responsive colour-changing actuators [73].

The mechanical response of oxidized nanofibrillated cellulose films, when exposed to water fluxes, was reported by Bettotti et al. [74]. These films reversibly transduce a water gradient into a fast and reversible bending movement. The bending movement follows the direction of the flux of water molecules and its rate is dependent on the magnitude of this flux [74]. Zhang et al. reported a cellulose stearoyl ester transparent free-standing and moisture-responsive film obtained after solvent-casting. The films presented bending movements and reversible shape changes 
(a)

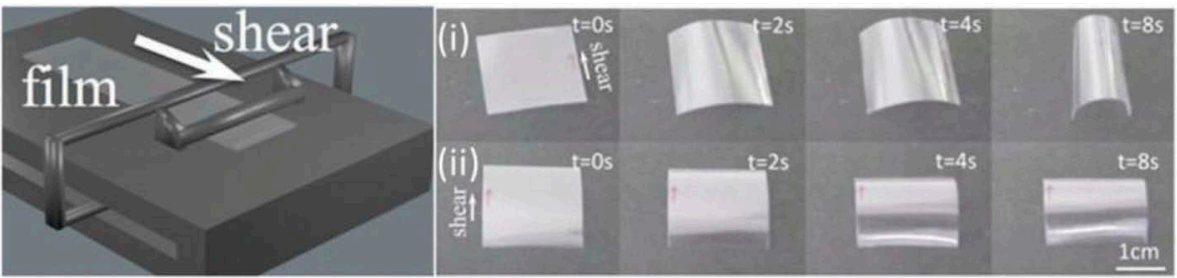

(b)

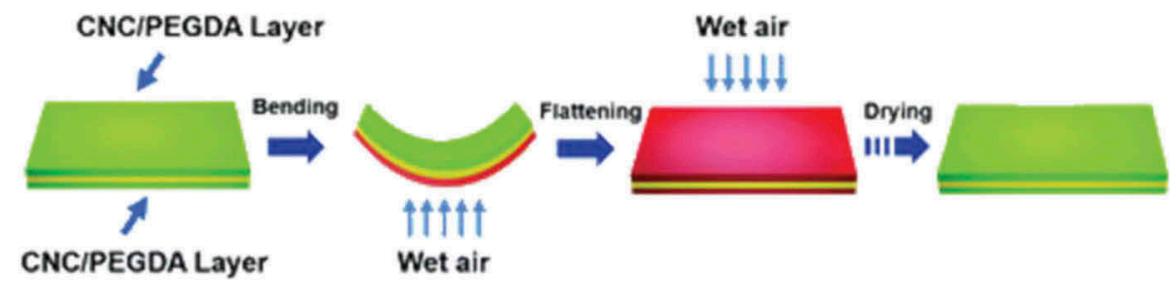

(c)

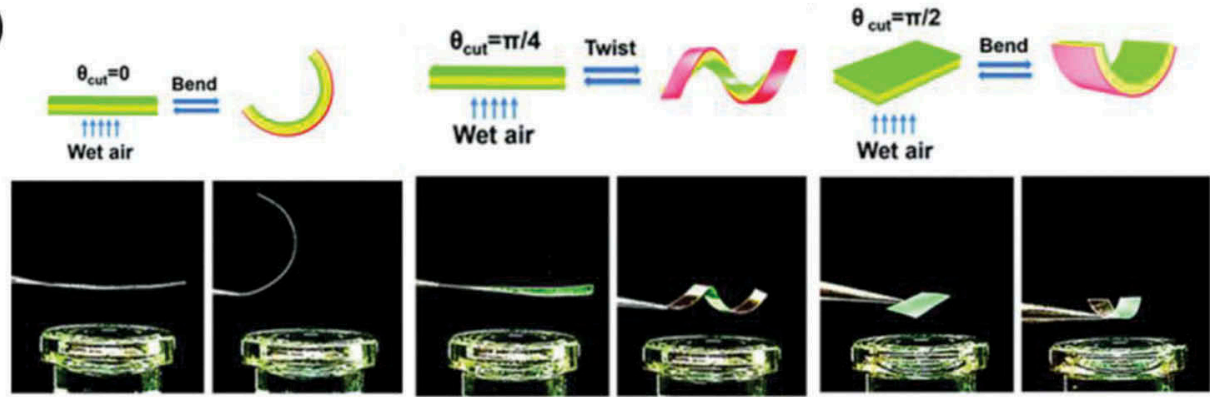

Figure 4. (Colour online) Cellulose-based systems responsive to moisture. (a) Schematic of the apparatus used to produce HPC shear casted films from lyotropic liquid crystalline solutions, with different thicknesses (range from $30 \mu \mathrm{m}$ to $100 \mu \mathrm{m}$ ) depending on the Gardner knife gap and speed. (i) Bending of the HPC free-standing films top surface and (ii) the film glass bottom surface exposed to water vapour, the arrows indicate the shear direction. Reproduced from [72] ® 2013, Springer Nature. (b) Schematic representation of water vapour-based actuation in a sandwich-structured nanocomposite film. (c) Scheme of a sandwich-structured composite film cut at different angles, which influence the shape-changing behaviour of the film (including bend and twist) and a corresponding image of the films cut at different angles (bottom images). Reproduced from [73] with permission of Royal Society of Chemistry in the format Journal/magazine via Copyright Clearance Center.

when exposed to water vapour [75]. If a bilayer film of cellulose stearoyl esters with different degrees of substitution on each side is produced, it is possible to obtain a combined responsiveness to moisture and temperature. It is also possible to adjust the minimal bending extent simply by changing the thickness of the cellulose stearoyl ester film [75].

The different spatial distributions of the constituents of the engineered cellulosic systems determine the shape and complexity of the deformation, similar to cellulose fibre orientation in plants, which dictates their shape modification as a response to external stimuli [64].

\section{Applications; inspiration coming from Nature. A new world of opportunities coming from cellulose-based materials}

Nature is a great inspiration for the design of new materials, the development of a cellulosic Bouligandtype structure, that is responsible for the intense colour in Polia condensata fruit, was reported by Kose et al.
[76]. A homogeneous, stretchable $\mathrm{CNC} /$ elastomer composite with a chiral nematic organization of $\mathrm{CNCs}$ was developed. The chiral nematic CNC elastomer (CNC E) can experience large deformations by applying mechanical stress, and rapidly return to its original shape when the stress is removed (Figure 5(a)). Once the composite is stretched, the robust chiral nematic structure unwinds into a pseudo-nematic arrangement. The SEM image of the CNC-E cross-section revealed a periodic structure (Figure 5(b)). When the CNC-E was observed between cross polarizers, brilliant interference colours that change with the mechanical stress applied (extension and contraction) were observed (Figure 5(c)) [76]. This stimuli-responsive material, with the reversible unwinding of the Bouligand structure, is a great candidate for applications in flexible optics and sensing. Cellulose nanocrystals are possible materials for the fabrication of these structures due to their natural chiral nematic self-assembly [77].

Transparent and flexible laminar composite cholesteric- and nematic-like CNC-based films were reported 


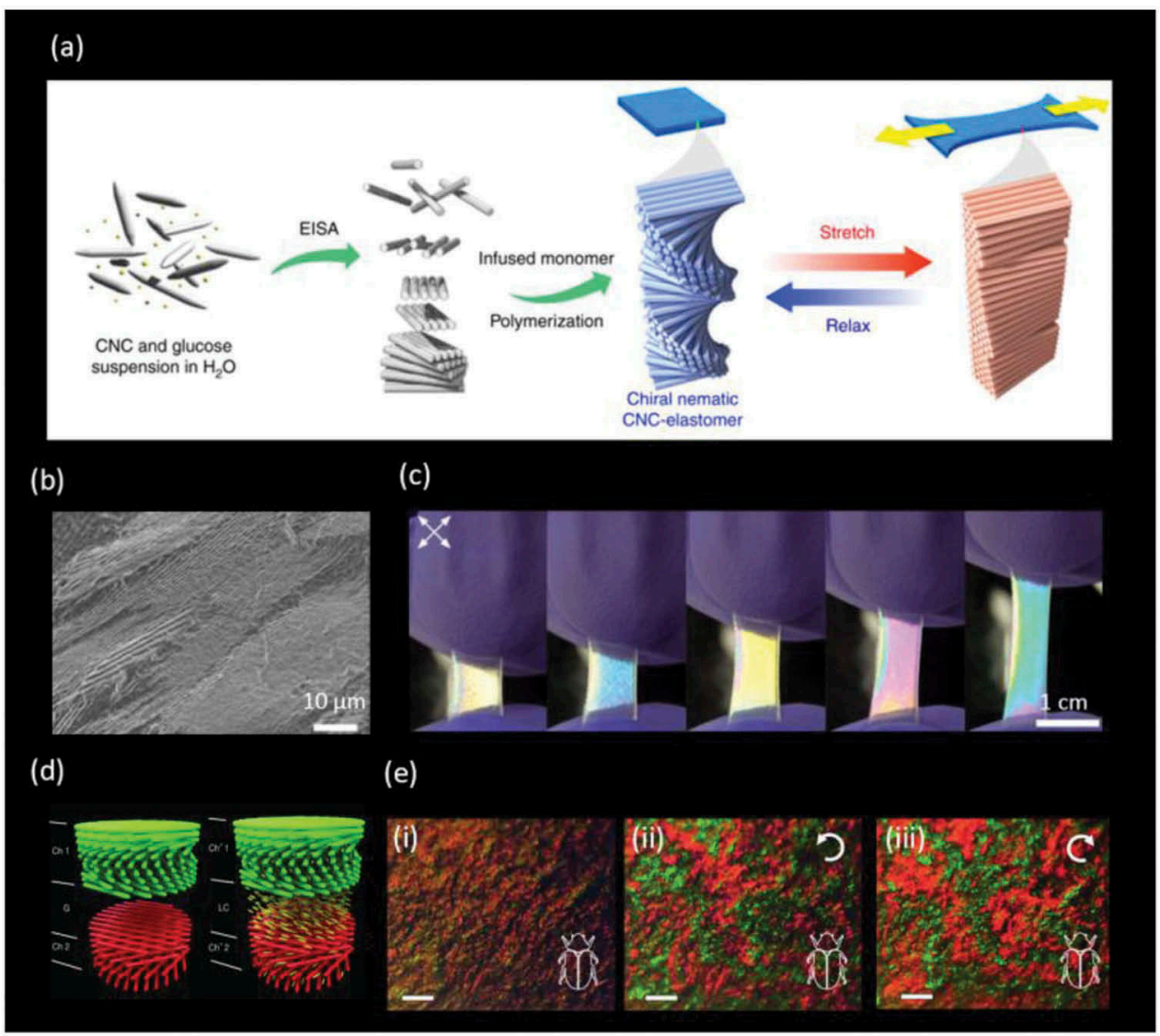

Figure 5. (Colour online) Tuning the colour of cellulose-based materials. (a) Schematic illustration of the preparation of the chiral nematic organization of CNCs and preparation process of CNC-E, (b) Cross-section SEM image of CNC-E film, (c) Photographs of CNCE stretching as viewed under crossed polarizers. The colour of the composite changes from white to blue to yellow to pink to green upon stretching. (Arrows in image indicate polarization axes of both linear polarizer and analyzer.) Reproduced from [76] under Creative Commons Attribution 4.0 International License. (d) scheme representing the cross-section near the gap, G, of a sandwich of two left cholesteric domains $\mathrm{Ch} 1$ and $\mathrm{Ch} 2$ with different pitches (left) and scheme showing the gap filled with the liquid crystal (5CB) sandwiched between the two left cholesteric domains (Ch'1 and Ch'2) (right). (e) Photographs of CNC and 5CB composite film observed through unpolarized white light (i) and circularly polarized light showing different reflection colours in LCP (ii) and RCP (iii) light channels as Plusiotis resplendens (silhouette in panels). Reproduced from [81] ๔ 2016 WILEY-VCH Verlag GmbH \& Co. KGaA, Weinheim.

by De La Cruz et al. [78]. A three-layer film with helicoidal and nematic-like organization of the cellulose nanocrystals mimics the cuticula of Plusiotis resplendens beetles. The birefringence of three-layer CNC film can be coarsely or finely tuned when bacterial or cotton CNC are used, respectively. This low-cost high reflective material can be used in colour filters or document security features [78].

The self-assembly process of coloured biomimetic cellulose films similar to Pollia condensata was reported by Dumanli et al. [79]. The fine-tuning of the colour of the film can be performed by carefully controlling the water evaporation. After quantification of the disorder within the sample, the authors stated that the colour fluctuations in these films are due to a non-uniform helical-pitch and not to chiral nematic director misalignment. These findings paved the way to the use of cellulose-based optic sensors [79]. It is also possible to combine the structural colouration with, for instance, shape memory effects, if the iridescent films of CNCs are embedded in a polydiolcitrate elastomer. This hybrid thermoresponsive chiral nematic photonic material can be used in a wide range of applications as multi-responsive device and sensors [80].

Freestanding CNC solid iridescent films, which reflect simultaneously right and left circularly polarized light were reported by Fernandes et al. [81]. These structures display similar optical characteristics to the cuticula of Plusiotis resplendens. To produce the cellulose-based photonic structure iridescent CNCs left-handed solid films were impregnated with 4-cyano-4'-pentylbiphenyl (5CB) nematic liquid crystal (LC) (Figure 5(d)). The light reflection of the structure was found to be tuned by temperature and by the application of an electrical field. 
The predominant colours of the film, under white light, was red and green, as shown in Figure 5(e) (i). The introduction of the liquid crystal on the CNC film increased the cellulose network cholesteric pitch, becoming the reflected green regions in the left circularly polarized (LCP), red in the right circularly polarized (RCP) channel and vice versa (Figure 5(e) (ii) and (iii)). The authors took advantage of the existence of gaps between left-hand cholesteric structures, to build the cellulose device, with response times in the order of milliseconds, with potential applications in photonics [81].

Another interesting cellulose-based photonic system is hydroxypropyl-cellulose (HPC)/water, which generates helical structures with Bragg-like reflections, determined by the helical pitch. The vivid colours observed with HPC/ water systems changed dynamically through the manipulation of the pitch, for example, by mechanical expansion or compression of the solutions $[50,82]$. The initial characteristic colour of the system (red, green or blue) is tuned by controlling the concentration of the polymer. Taking into consideration this characteristic and the variation of the cholesteric pitch with pressure, Liang et al. [12] manufactured a meter-scale HPC/water laminate. These HPC stimuli-responsive photonic films present a mechanochromic pressure response that enables the recording of pressure profiles generated by foot-prints in real time. This approach presents a great potential for cost-effective largearea mapping applications [12].

Inspired by botanical systems, such as tendrils, leaves or flowers, Gladman et al. printed a composite NCC hydrogel [83]. The encoded architecture is achieved by a combination of materials and geometry that can be controlled in space and time. The hydrogel composite ink used for $4 \mathrm{D}$ printing is composed of stiff cellulose fibrils embedded in a soft acrylamide matrix. The anisotropic swelling of the material allows precise control over the structure curvature. The hydrogel composite ink formulation can be extended to a broad range of matrices and anisotropic fillers, and if combined with flow-induced anisotropy will allow the production of reconfigurable materials with tunable functionality. This methodology paves the way for the creation of new shape-shifting architectures for several applications such as tissue engineering, biomedical devices and soft robotics [83].

\section{Summary and outlook}

Solid structures resembling ordered cholesteric phases are present in cellulosic structures, which are found in the cell wall of the tissues of Plants. The nematic chiral phase organization assures not only the mechanical properties of the Plant but is also responsible for the structural colours displayed by some fruits, leaves and flowers. Quite fascinating and unique is to take the white cotton and 'decompose' it into a pallet of vivid cellulosebased structural colours, which goes from violet to red.

It is interesting to note that helicoidal responsive structures, imprinted by some plants, remain active in dead tissues even after leaving the plant. These observations are a source of inspiration for producing cellulosic materials with features completely different from those currently known for the use of cellulosic materials, which are mainly used for packaging and paper production.

Considering the replacement of plastics by materials of plant origin, it is interesting to think that cellulose-based materials can not only replace the polyolefins, used for conventional applications, but it opens paths to hitherto not explored applications. In this context, it worth noting the production of materials with structural colours as well as motors, which are actuated by moisture gradients. Although the complicated structures produced by plants are not possible to mimic, the solid structures, obtained from cellulosic liquid crystalline structures, are a source of inspiration for the manufacturing of functional materials, from the most abundant polymer on our planet.

\section{Acknowledgments}

The authors acknowledge National Funds through FCT Portuguese Foundation for Science and Technology and FCT/MCTES. Under projects numbers UID/CTM/50025/ 2019, PTDC/FIS-NAN/0117/2014, PTDC/CTM-BIO/6178/ 2014, M-ERA-NET2/0007/2016 (CellColor) and PTDC/ CTM/30529/2017. A.P.A. acknowledge the Minister of Science, Technology, and Higher Education for National Funds, European Social Funds, and FCT for the fellowship with reference SFRH/BD/115567/2016.

\section{Disclosure statement}

No potential conflict of interest was reported by the authors.

\section{Funding}

This work is funded by National Funds through FCT Portuguese Foundation for Science and Technology and FCT/MCTES. Under projects numbers UID/CTM/50025/ 2019, PTDC/FIS-NAN/0117/2014, PTDC/CTM-BIO/6178/ 2014, M-ERA-NET2/0007/2016 (CellColor) and PTDC/ CTM/30529/2017. A.P.A. acknowledge the Minister of Science, Technology, and Higher Education for National Funds, European Social Funds, and FCT for the fellowship with reference SFRH/BD/115567/2016.

\section{ORCID}

Ana P. C. Almeida (D) http://orcid.org/0000-0003-4984-0759 João P. Canejo (D) http://orcid.org/0000-0002-5361-7368 
Pedro L. Almeida (D) http://orcid.org/0000-0001-7356-8455 Maria Helena Godinho (D) http://orcid.org/0000-0002-97605983

\section{References}

[1] Ball P. Nature's palette: the science of plant color. Nature. 2007;449:982.

[2] Vukusic P, Sambles JR. Photonic structures in biology. Nature. 2003;424:852-855.

[3] Burresi M, Cortese L, Pattelli L, et al. Bright-white beetle scales optimise multiple scattering of light. Sci Rep. 2014;4:6075.

[4] Parker AR. 515 million years of structural colour. J Opt a-Pure Appl Op. 2000;2:R15-R28.

[5] Vignolini S, Rudall PJ, Rowland AV, et al. Pointillist structural color in Pollia fruit. Proc Natl Acad Sci U S A. 2012;109:15712-15715.

[6] Fernandes SN, Geng Y, Vignolini S, et al. Structural color and iridescence in transparent sheared cellulosic films. Macromol Chem Phys. 2013;214:25-32.

[7] Morris RB. Iridescence from diffraction structures in the wing scales of callophrys rubi, the green hairstreak. J Entomol Ser A Gen Entomol. 1975;49:149-154.

[8] Doucet SM, Meadows MG. Iridescence: a functional perspective. J R Soc Interface. 2009;6:S115-S32.

[9] Gruson H, Andraud C, de Marcillac WD, et al. Quantitative characterization of iridescent colours in biological studies: a novel method using optical theory. Interface Focus. 2019;9.

[10] Prelog V. Chirality in Chemistry. J Mol Catal. 1976;1:159-172.

[11] Murugesan YK, Rey AD. Modeling textural processes during self-assembly of plant-based chiral-nematic liquid crystals. Polymers. 2010;2:766-785.

[12] Liang H-L, Bay MM, Vadrucci R, et al. Roll-to-roll fabrication of touch-responsive cellulose photonic laminates. Nat Commun. 2018;9:4632.

[13] Nguyen TD, Sierra E, Eguiraun H, et al. Iridescent cellulose nanocrystal films: the link between structural colour and Bragg's law. Eur J Phys. 2018;39.

[14] Seo HJ, Lee SS, Noh J, et al. Robust photonic microparticles comprising cholesteric liquid crystals for anti-forgery materials. J Mater Chem C. 2017;5:7567-7573.

[15] Almeida APC, Canejo JP, Fernandes SN, et al. Cellulose-based biomimetics and their applications. Adv Mater. 2018;30:1703566.

[16] Dumanli AG, van der Kooij HM, Kamita G, et al. Digital color in cellulose nanocrystal films. Acs Appl Mater Inter. 2014;6:12302-12306.

[17] Fernandes SN, Almeida PL, Monge N, et al. Mind the microgap in iridescent cellulose nanocrystal films. Adv Mater. 2017;29:1603560.

[18] Goodby JW. Symmetry and Chirality in Liquid Crystals. In: Demus DJG, Gray GW, Spiess H, et al., editors. Handbook of liquid crystals set. Weinheim: WILEY-VCH; 1998. p. 115-132.

[19] Neville AC, Caveney S. Scarabaeid beetle exocuticle as an optical analogue of cholesteric liquid crystals. Biol Rev. 1969;44:531-562.
[20] Neville AC. Biology of fibrous composites: development beyond the cell membrane. Cambridge, UK: Cambridge University Press; 1993.

[21] Bouligand Y. Comparaison De Certains Materiels Biologiques a La Structure Des Cristaux Liquides Cholesteriques. J Microsc-Oxford. 1967;6:A41-+.

[22] Bouligand Y. Twisted fibrous arrangements in biological-materials and cholesteric mesophases. Tissue Cell. 1972;4:189.

[23] Bouligand Y. Study of mesomorphic textures .2. Polygonal fields in cholesteries. J Phys-Paris. 1972;33:715-736.

[24] Bouligand Y. Liquid crystals and biological morphogenesis: ancient and new questions. Cr Chim. 2008;11:281-296.

[25] Bouligand Y, Livolant F. The organization of cholesteric spherulites. J Phys-Paris. 1984;45:1899-1923.

[26] Bouligand Y. Sur Une Disposition Fibrillaire Torsadee Commune a Plusieurs Structures Biologiques. Cr Hebd Acad Sci. 1965;261:4864-4867.

[27] Bouligand Y. Sur L'Existence De "Pseudomorphoses Cholestériques" Chez Divers Organismes Vivants. J Phys Colloques. 1969;30:C4-90-C4-103.

[28] Roland JC, Reis D, Vian B, et al. Morphogenesis of plant-cell walls at the supramolecular level - internal geometry and versatility of helicoidal expression. Protoplasma. 1987;140:75-91.

[29] Graham RM, Lee DW, Norstog K. Physical and Ultrastructural Basis of Blue Leaf Iridescence in Two Neotropical Ferns. Am J Bot. 1993;80:198-203.

[30] Gould KS, Lee DW. Physical and ultrastructural basis of blue leaf iridescence in four Malaysian understory plants. Am J Bot. 1996;83:45-50.

[31] Vignolini S, Gregory T, Kolle M, et al. Structural colour from helicoidal cell-wall architecture in fruits of Margaritaria nobilis. J $\mathrm{R}$ Soc Interface. 2016;13:20160645.

[32] Reis D. Cholesteric-like pattern in plant-cell walls different expressions. Mol Cryst Liq Cryst. 1987;153:43-53.

[33] Rånby BG. Fibrous macromolecular systems. Cellulose and muscle. The colloidal properties of cellulose micelles. Discuss Faraday Soc. 1951;11:158-164.

[34] Habibi Y, Lucia LA, Rojas OJ. Cellulose nanocrystals: chemistry, self-assembly, and applications. Chem Rev. 2010;110:3479-3500.

[35] Mukherjee SM, Woods HJ. X-ray and electron microscope studies of the degradation of cellulose by sulphuric acid. Biochim Biophys Acta. 1953;10:499-511.

[36] Emerton HW, Wrist PE, Sikorski J, et al. Electronmicroscopy of degraded cellulose fibres. J Text Inst Trans. 1952;43:T563-T4.

[37] Marchessault RH, Morehead FF, Walter NM. Liquid crystal systems from fibrillar polysaccharides. Nature. 1959;184:632-633.

[38] Klemm D, Kramer F, Moritz S, et al. Nanocelluloses: a new family of nature-based materials. Angew Chem. 2011;50:5438-5466.

[39] Matos Ruiz M, Cavaillé JY, Dufresne A, et al. Processing and characterization of new thermoset nanocomposites based on cellulose whiskers. Compos Interfaces. 2000;7:117-131. 
[40] Revol JF, Bradford H, Giasson J, et al. Helicoidal self-ordering of cellulose microfibrils in aqueous suspension. Int J Biol Macromol. 1992;14:170-172.

[41] Fleming K,G, Gray D, Matthews S. Cellulose crystallites. Chemistry. 2001;7(9):1831-1835.

[42] Fried F, Gilli JM, Sixou P. The cholesteric pitch in lyotropic solutions of a semi-rigid macromolecule: hydroxypropyl-cellulose. Mol Cryst Liq Cryst. 1983;98:209-221.

[43] Zugenmaier P. Cellulosic liquid crystals. In: Demus DJG, Gray GW, Spiess $\mathrm{H}$, et al., editors. Handbook of liquid crystals. Vol. 3. Weinheim: WILEY-VCH; 1998. p. 453-482.

[44] Chanzy H, Peguy A, Chaunis S, et al. Oriented cellulose films and fibers from a mesophase system. J Polym Sci. 1980;18:1137-1144.

[45] Navard P, Haudin J-M. Rheololgy of mesomorphic solutions of cellulose. Br Polym J. 1980;12:174-178.

[46] Patel DL, Gilbert RD. Lyotropic mesomorphic formation of cellulose in trifluoroacetic acid-chlorinatedalkane solvent mixtures at room temperature. J Polym Sci. 1981;19:1231-1236.

[47] McCormick CL, Callais PA, Hutchinson BH. Solution studies of cellulose in lithium chloride and N,N-dimethylacetamide. Macromolecules. 1985;18:2394-2401.

[48] Werbowyj RS, Gray DG. Liquid crystalline structure in aqueous hydroxypropyl cellulose solutions. Mol Cryst Liq Cryst. 1976;34:97-103.

[49] Tseng S-L, Valente A, Gray DG. Cholesteric liquid crystalline phases based on (acetoxypropyl)cellulose. Macromolecules. 1981;14:715-719.

[50] Godinho MH, Gray DG, Pieranski P. Revisiting (hydroxypropyl) cellulose (HPC)/water liquid crystalline system. Liq Cryst. 2017;44:2108-2120.

[51] Bheda J, Fellers JF, White JL. Phase behavior and structure of liquid crystalline solutions of cellulose derivatives. Colloid Polym Sci. 1980;258:1335-1342.

[52] Flory PJ, Gee G Statistical thermodynamics of semi-flexible chain molecules. Proceedings of the Royal Society of London Series A Mathematical and Physical Sciences. 1956;234. p. 60-73.

[53] Kuhn W. Beziehungen zwischen Molekülgröße, statistischer Molekülgestalt und elastischen Eigenschaften hochpolymerer Stoffe. Kolloid-Zeitschrift. 1936;76:258-271.

[54] Shimamura K, White JL, Fellers JF. Hydroxypropylcellulose, a thermotropic liquid crystal: characteristics and structure development in continuous extrusion and melt spinning. J Appl Polym Sci. 1981;26:2165-2180.

[55] Gray DG. Liquid crystalline cellulose derivatives. Cellulose Conference. 24 May 1982.

[56] Mitov M. Cholesteric liquid crystals in living matter. Soft Matter. 2017;13:4176-4209.

[57] Emons AMC, Mulder BM. How the deposition of cellulose microfibrils builds cell wall architecture. Trends Plant Sci. 2000;5:35-40.

[58] Tan T, Ribbans B. A bioinspired study on the compressive resistance of helicoidal fibre structures. Proc Math Phys Eng Sci. 2017;473:20170538-.Epub 10/11.
[59] Canejo JP, Fernandes SN, Godinho MH, et al. Liquid fibres and their networks from cellulose-based liquid crystalline solutions. Liq Cryst. 2018;45:1987-1995.

[60] Dawson C, Vincent JFV, Rocca A-M. How pine cones open. Nature. 1997;390:668.

[61] Song K, Yeom E, Lee SJ. Real-time imaging of pulvinus bending in Mimosa pudica. Sci Rep. 2014;4:6466.

[62] Forterre Y, Skotheim JM, Dumais J, et al. How the Venus flytrap snaps. Nature. 2005;433:421-425.

[63] Lydon J. Silk and Fibers, Collagen. In: Goodby JW, Raynes CT,P, Gleeson H, et al., editors. Handbook of Liquid Crystals. Vol. 7. Weinheim: Wiley-VCH; 2014. p. 1-53.

[64] Li S, Wang KW. Plant-inspired adaptive structures and materials for morphing and actuation: a review. Bioinspir Biomim. 2016;12:011001.

[65] Poppinga S, Zollfrank C, Prucker O, et al. Toward a new generation of smart biomimetic actuators for architecture. Adv Mater. 2018;30:1703653.

[66] Abraham Y, Tamburu C, Klein E, et al. Tilted cellulose arrangement as a novel mechanism for hygroscopic coiling in the stork's bill awn. J R Soc Interface. 2012;9:640-7.Epub 08/24.

[67] Zhao C, Liu QP, Ren LQ, et al. A 3D micromechanical study of hygroscopic coiling deformation in Pelargonium seed: from material and mechanics perspective. J Mater Sci. 2017;52:415-430.

[68] Abraham Y, Elbaum R. Hygroscopic movements in Geraniaceae: the structural variations that are responsible for coiling or bending. New Phytol. 2013;199:584-594.

[69] Jung W, Kim W, Kim HY. Self-burial mechanics of hygroscopically responsive awns. Integr Comp Biol. 2014;54:1034-1042.

[70] Elbaum R, Gorb S, Fratzl P. Structures in the cell wall that enable hygroscopic movement of wheat awns. J Struct Biol. 2008;164:101-107.

[71] Almeida APC, Querciagrossa L, Silva PES, et al. Reversible water driven chirality inversion in cellulose-based helices isolated from Erodium awns. Soft Matter. 2019;15:2838-2847.

[72] Geng Y, Almeida PL, Fernandes SN, et al. A cellulose liquid crystal motor: a steam engine of the second kind. Sci Rep. 2013;3:1028.

[73] $\mathrm{Wu} \mathrm{T}, \mathrm{Li} \mathrm{J}, \mathrm{Li}$ J, et al. A bio-inspired cellulose nanocrystal-based nanocomposite photonic film with hyper-reflection and humidity-responsive actuator properties. J Mater Chem C. 2016;4:9687-9696.

[74] Bettotti P, Maestri CA, Guider R, et al. Dynamics of hydration of nanocellulose films. Adv Mater Interfaces. 2016;3:1500415.

[75] Zhang K, Geissler A, Standhardt M, et al. Moistureresponsive films of cellulose stearoyl esters showing reversible shape transitions. Sci Rep. 2015;5:11011.

[76] Kose O, Tran A, Lewis L, et al. Unwinding a spiral of cellulose nanocrystals for stimuli-responsive stretchable optics. Nat Commun. 2019;10:510.

[77] Natarajan B, Gilman JW. Bioinspired Bouligand cellulose nanocrystal composites: a review of mechanical properties. Philos Trans R Soc A Math Phys Eng Sci. 2018;376:20170050. 
[78] De La Cruz JA, Liu Q, Senyuk B, et al. Cellulose-based reflective liquid crystal films as optical filters and solar gain regulators. ACS Photonics. 2018;5:2468-2477.

[79] Dumanli AG, Kamita G, Landman J, et al. Controlled, bio-inspired self-assembly of cellulose-based chiral reflectors. Adv Opt Mater. 2014;2:646-650.

[80] Espinha A, Guidetti G, Serrano MC, et al. Shape memory cellulose-based photonic reflectors. Acs Appl Mater Inter. 2016;8:31935-31940.
[81] Fernandes SN, Almeida PL, Monge N, et al. Mind the microgap in iridescent cellulose nanocrystal films. Adv Mater. 2017;29:1603560.

[82] Kamita G, Frka-Petesic B, Allard A, et al. Biocompatible and sustainable optical strain sensors for large-area applications. Adv Opt Mater. 2016;4:1950-1954.

[83] Sydney Gladman A, Matsumoto EA, Nuzzo RG, et al. Biomimetic 4D printing. Nat Mater. 2016;15:413. 